\title{
Perioperative complications after transoral endoscopic thyroidectomy vestibular approach: a retrospective audit study
}

\author{
Kuo-Chuan Hung', Li-Kai Wang', Kuo Mao Lan', Jen-Yin Chen ${ }^{1,2^{*}}$
}

'Department of Anesthesiology, Chi Mei Medical Center, Tainan, Taiwan

2Department of the Senior Citizen Service Management, Chia Nan University of Pharmacy and Science, Tainan, Taiwan

\section{Background}

Transoral endoscopic thyroidectomy vestibular approach (TOETVA) has been reported to be safe with minimal trauma and superior cosmetic outcome. Our retrospective audit study primarily aimed at analyzing perioperative complications in patients undergoing TOETVA at an Asian tertiary referral center.

\section{Methods}

The demographic and anthropometric data as well as perioperative and postoperative complications of 124 patients undergoing TOETVA at Chi Mei medical center from October 2015 to March 2017 (i.e., 18 months) were retrospectively reviewed. To determine the effect of operative experience on the incidence of complications, the study period was divided into two phases (i.e., Phase 1 and Phase 2) with the study patients equally divided into two groups after the first introduction of TOETVA.

\section{Results}

Totally 124 patients (110 females and 14 males) were studied. The median age was $46.5 \pm 12$ years (Table 1 ). The incidence of major perioperative complications was in the order of massive subcutaneous emphysema (58.1\%), difficult breathing with or without stridor (9.7\%), tracheal tube cuff rupture (3.2\%), and cervical hematoma (1.6\%) (Table 2). All patients were discharged from hospital without long-term sequelae. The operating time $(p=0.005)$, anesthesia time $(p=0.024)$, and complications rate $(p<0.001)$ decreased significantly in Phase 1 compared with that in Phase 2.

Table 1. Baseline demographic, anthropometric, and disease characteristics of study subjects $(n=124)$

\begin{tabular}{lcccc} 
& $\begin{array}{c}\text { Total } \\
\mathrm{n}=124\end{array}$ & $\begin{array}{c}\text { Phase 1 } \\
\mathrm{n}=62\end{array}$ & $\begin{array}{c}\text { Phase 2 } \\
\mathrm{n}=62\end{array}$ & $p$ value \\
\hline Gender (female) & $110(88.7)$ & $52(83.9)$ & $58(93.5)$ & 0.154 \\
Age (years) & $46.5 \pm 12$ & $45.4 \pm 12.4$ & $47.6 \pm 11.5$ & 0.309 \\
Height $(\mathrm{cm})$ & $159.3 \pm 7.1$ & $160.3 \pm 7.7$ & $158.3 \pm 6.4$ & 0.111 \\
Weight $(\mathrm{kg})$ & $59.8 \pm 11.7$ & $60.6 \pm 13$ & $59.1 \pm 10.3$ & 0.453 \\
Body mass index $\left(\mathrm{kg} / \mathrm{m}^{2}\right)$ & $23.5 \pm 3.7$ & $23.4 \pm 3.8$ & $23.5 \pm 3.6$ & 0.889 \\
ASA classification & & & & 0.126 \\
I-II & $112(90.3)$ & $59(95.2)$ & $53(85.5)$ & \\
Pathologic diagnosis & & & & 0.158 \\
Multinodular goiter & $90(72.6)$ & $49(79)$ & $41(66.1)$ & \\
Others & $34(27.4)$ & $13(21)$ & $21(33.9)$ & \\
Gland size $(\mathrm{cm})^{\ddagger}$ & $3.3 \pm 1.6$ & $3.4 \pm 1.6$ & $3.2 \pm 1.7$ & 0.5 \\
\hline
\end{tabular}

TOETVA implementation; §Others include Graves' disease, papillary carcinoma,

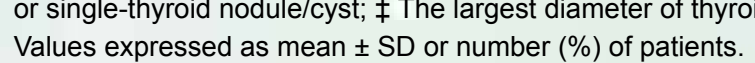

Table 2. Perioperative complications of study subjects

\begin{tabular}{lcccc}
\hline \multicolumn{1}{|c}{ Variables } & $\begin{array}{c}\text { Total } \\
\mathrm{n}=124\end{array}$ & $\begin{array}{c}\text { Phase 1 } \\
\mathrm{n}=62\end{array}$ & $\begin{array}{c}\text { Phase 2 } \\
\mathrm{n=62}\end{array}$ & p value \\
\hline Total incidence of complications $\ddagger$ & 91 & 57 & 34 & $<0.001$ \\
\hline Massive subcutaneous emphysema & $72(58.1)$ & $44(71)$ & $28(45.2)$ & 0.004 \\
\hline Difficult breathing with or without stridor & $12(9.7)$ & $8(12.9)$ & $4(6.5)$ & 0.224 \\
\hline Rupture of endotracheal tube cuff & $4(3.2)$ & $4(6.5)$ & 0 & 0.119 \\
\hline Cervical hematoma & $2(1.6)$ & $1(1.6)$ & $1(1.6)$ & 1 \\
\hline Nasal-ala pressure sore & $1(0.8)$ & 0 & $1(1.6)$ & 1 \\
\hline Pneumomediastinum or pneumothorax & 0 & 0 & 0 & NCs \\
\hline
\end{tabular}

*Values expressed as mean \pm SD or number (\%) of patients; §NC: Not calculated.

\section{Conclusion}

Although perioperative complications were not uncommon in patients undergoing TOETVA, most were minor without long-term sequelae. Furthermore, complication rate decreased with increasing experience. Our findings may provide insights into the prevention of TOETVA-associated perioperative complications.

\section{References}

1. Witzel K, Hellinger A, Kaminski C, Benhidjeb T. Endoscopic thyroidectomy: the transoral approach. Gland Surg. 2016; 5:336-41.

2. Lombardi CP, Raffaelli M, Princi P, De Crea C, Bellantone R. Video-assisted thyroidectomy: report on the experience of a single center in more than four hundred cases. World J Surg. 2006; 30: 794-800.

3. Wang C, Zhai H, Liu W, Li J, Yang J, Hu Y, Huang J, Yang W, Pan Y, Ding H. Thyroidectomy: a novel endoscopic oral vestibular approach. Surgery. 2014; 155 : 33-8. 